More broadly, understanding and potentially influencing public opinion will be important components of any strategies to combat COVID-19 and prevent future pandemics ${ }^{10}$. This global problem requires global solutions, and our survey indicates that the redistribution of some pre-purchased vaccines to countries most in need has public support.

\section{Data Availability}

Data are available from the authors upon request.

\section{Editorial note: This article has been peer-reviewed.}

\section{Philip M. Clarke (D) ${ }^{凶}$, Laurence S. J. Roope (iD) Peter John Loewen ${ }^{2}$, Jean-Francois Bonnefon ${ }^{3}$, Alessia Melegaro (D) 4 , Jorge Friedman ${ }^{5}$, Mara Violato ${ }^{1}$, Adrian Barnett ${ }^{6}$ and Raymond Duch (D) \\ ${ }^{1}$ Health Economics Research Centre, Nuffield Department of Population Health, University of Oxford, Oxford, UK. ${ }^{2}$ Department of Political Science and the Munk School of Global Affairs \& Public Policy, University of Toronto, Toronto, Ontario, Canada. ${ }^{3}$ Toulouse School of Economics, Toulouse, France. ${ }^{4}$ Dondena Centre for Research on Social}

Dynamics and Public Policy, Department of Social and Political Sciences, Bocconi University, Milan, Italy. ${ }^{5}$ Facultad de Administración y Economía, Universidad de Santiago de Chile, Santiago, Chile. ${ }^{6}$ Queensland University of Technology, Brisbane, Queensland, Australia. ${ }^{7}$ Nuffield College, University of Oxford, Oxford, UK.

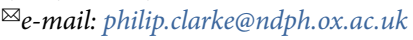

Published online: 30 March 2021

https://doi.org/10.1038/s41591-021-01322-9

References

1. Mullard, A. Nature https://www.nature.com/articles/d41586-02003370-6 (2020).

2. Nature 589, 170 (2021).

3. Kumar, S., Quinn, S. C., Kim, K. H. \& Hilyard, K. M. PLoS One 7, e33025 (2012).

4. Duch, R. et al. Preprint at https://www.medrxiv.org/content/10.11 01/2021.01.31.21250866v1 (2021).

5. Duke Global Health Innovation Center. https:// launchandscalefaster.org/COVID-19 (accessed 9 March 2020).

6. Çakmakl, C., Demiralp, S., Kalemli-Özcan, S., Yeşiltaş, S. \& Ylldırım, M.A. National Bureau of Economic Research https://doi. org/10.3386/w28395 (2021).

7. Hafner, M., Yerushalmi, E., Fays, C., Dufresne, E. \& Van Stolk, C. RAND Corporation https://www.rand.org/pubs/research_reports/ RRA769-1.html (2020).

8. Rigby, J. \& Newey, S. The Telegraph https://www.telegraph. co.uk/global-health/science-and-disease/norway-share-covid19-vaccine-poorer-countries-time-protecting/ (20 January 2021).

9. Hogan, A.B. et al. Imperial College London https://doi. org/10.25561/82822 (25 September 2020).

10. Bonell, C. et al. J. Epidemiol. Community Health 74, 617-619 (2020).

\section{Acknowledgements}

The research was supported by funding from the National Institute for Health Research (NIHR) Oxford Biomedical Research Centre and the Department of Health and Social Care for evaluation of the COVID-19 Oxford Vaccine Trial. The views expressed are those of the author(s) and not necessarily those of the NHS, the NIHR or the Department of Health and Social Care. We acknowledge the support of the Office of the Dean of the Faculty of Arts \& Science at the University of Toronto. J.-F.B. acknowledges support from the grant ANR-17-EURE-0010 Investissements d'Avenir. J.F. received funding from the University of Santiago project Dicyt USA1899. A.M. acknowledges support from the Italian Ministry of Education Progetti di Rilevante Interesse Nazionale, grant number 20177BRJXS M.V. receives funding from the NIHR Applied Research Collaboration Oxford and Thames Valley at Oxford Health NHS Foundation Trust. R.D. acknowledges support from the grant FONDECYT No1201397. The survey study was approved by the University of Oxford Medical Sciences Interdivisional Research Ethics Committee (approval ID: R72328/RE001).

Author contributions

P.M.C. and R. D., overall design of the CANDOUR study; L.S.J.R., P.J.L. and M.V., design of the questionnaire; J.-F.B., A.M. and J.F., interpretation of the results; and A.B., statistical analysis. All authors contributed to the manuscript and approved the final version.

\title{
Improving patient care through the development of a 5G-powered smart hospital
}

To the Editor-In 2018, Guangdong Second Provincial General Hospital (GD2H) started incorporating artificial intelligence (AI) into hospital management and operations, including patient registration and triage, diagnosis aids, health-record organization, digital payment, and the transportation of operating-room supplies $^{1,2}$. Due to the limitations of big-data sharing via the current $4 \mathrm{G}$ hospital network, practical applications of AI cannot be closely connected throughout the hospital, which compromises efficiency and reduces patient satisfaction. For example, if a patient is waiting for emergency surgery in the operating room after a magnetic resonance imaging scan, the surgeons can proceed only until the image files and reports are transferred to the system in the operating room, which takes time and causes delays.
To address this issue, GD2H recently announced the building of a comprehensive smart hospital in conjunction with Huawei, using $5 \mathrm{G}$ technology that features low latency, high capacity, increased bandwidth and a wireless nature ${ }^{3}$. The $5 \mathrm{G}$ hospital has attracted worldwide attention because of the potential for fundamentally changing how hospitals operate. By using $5 \mathrm{G}$ in combination with cloud storage and AI, the comprehensive $5 \mathrm{G}$ smart hospital will cover areas of healthcare, teaching and training, research, and management, with $5 \mathrm{G}$ technology applied both within and outside the hospital, including ambulance, outpatient and inpatient services, and the operating room. The $5 \mathrm{G}$ smart hospital has several potential benefits, as outlined below.

In 2019, GD2H started to guide complex surgery conducted in remote hospitals, connected live via $5 \mathrm{G}$, that allowed the operating room to be turned into a classroom ${ }^{4}$. Since then, GD2H has continued to explore ways that $5 \mathrm{G}$ could overcome the problem of real-time data sharing due to distance and volume of data. GD2H has now equipped its ambulances with a portable computerized tomography scanner, an electrocardiogram and an echocardiogram machine, as well as first-aid supplies. Once a patient enters the ambulance, the use of $5 \mathrm{G}$ allows real-time data on rapid assessment, with examinations and diagnoses, monitoring, and initial treatments transmitted to the hospital system simultaneously. If necessary, a multidisciplinary team can arrive within minutes for consultation and decision-making, while the emergency room is made ready to receive the patient. The 5G-powered ambulance as a mini-hospital will shorten the time from 


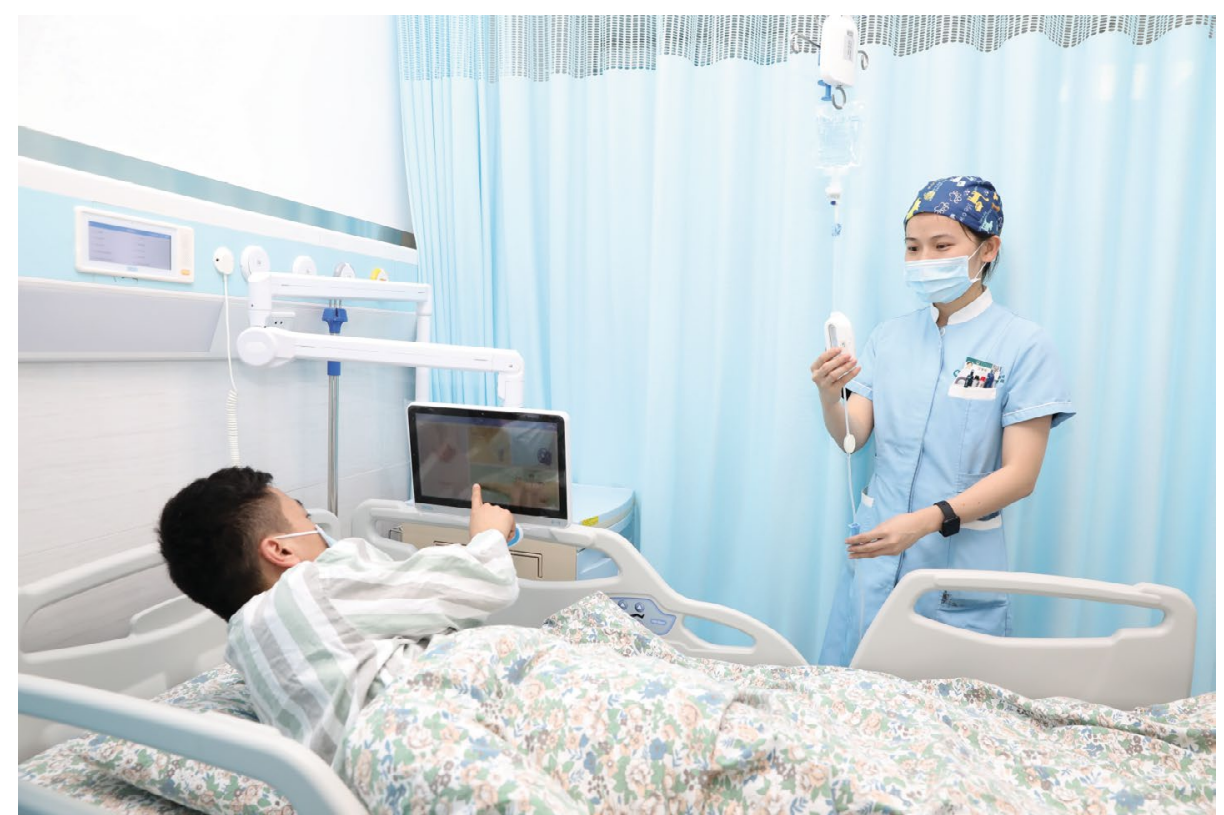

A nurse initiating a smart infusion device for intravenous therapy while the patient checks his treatment plan on the bedside smart touchscreen. Credit: GD2H (taken by Haoshu He)

disease onset to treatment received, which should improve the survival of patients and achieve better outcomes.

The smart hospital at GD2H has applied 5G widely in the wards, which also make use of wearable trackers, smart touchscreens, robots for supply delivery and cleaning, infusion devices, and real-time monitoring and warning systems. Infusion devices transmit data on the speed and time remaining to the monitoring system automatically, while a warning signal is sent to the operating desk and the nurse's wearable tracker, worn on the wrist, if anything requires clinical attention (Fig. 1). This is especially appreciated by patients with sleep problems, because the nurse can handle the infusion via remote tracking, without interrupting the patient's sleep.

Patients in the wards can also receive remote consultations and monitor their own treatment. Patients can access all the data on examinations, treatments and expenses either from bedside touchscreens or from the smartphone app DingBei Doctor, which was developed by GD2 $\mathrm{H}^{5}$. Accessing online consultations and discussions should improve inpatients' satisfaction, facilitate management of their own health conditions and, ultimately, improve patient-centered outcomes.
Experiences with 5G smart hospitals such as GD2H may provide lessons for internet hospitals. The internet hospital is a fully digital platform that provides healthcare services and has the potential to be a telehealth model for healthcare provision and consumption in China. Internet hospitals aim to alleviate the disparity in healthcare resources in different parts of China, and to satisfy the emerging needs of patients, who want to receive medical care through an online platform, without the need for travel ${ }^{6}$. The internet hospital also provides an opportunity for controlling nosocomial infections, especially during the COVID-19 pandemic ${ }^{7}$. Patients can receive a contactless professional consultation and medical advice before visiting a hospital and, if needed, they can request home delivery of treatments after receiving an electronic prescription. The unavailability of big-data sharing is a key barrier to the efficiency and usage of an internet hospital ${ }^{8}$, so the use of $5 \mathrm{G}$ may be a potential solution to increase the use of internet hospitals.

In the future, we expect that the use of $5 \mathrm{G}$ in conjunction with AI will help with data-driven hospital management and decision-making, due to the extensive information available instantaneously, combined with a decision support system.
Such rapid decision-making would have been very helpful at the start of the COVID19 pandemic, when public panic led to a surge of patients into hospitals.

Other uses of the $5 \mathrm{G}$ smart hospital include hospital security, training for novice practitioners and researchers, patient self-management, and student teaching. There are also challenges, including public acceptance of $5 \mathrm{G}$, the cost of infrastructure building, data security and privacy protections. The initiation of a 5G-powered smart hospital at GD2H provides an exploratory platform for addressing these concerns. as well as for exploring how this technology can enhance patient outcomes and instill a culture of evidence-based decision-making.

Guowei Li ${ }^{1}, 2$, Wanmin Lian ${ }^{3}$, Hongying $\mathrm{Qu}^{1}$, Ziyi Li (D)', Qiru Zhou ${ }^{4}$ and Junzhang Tian ${ }^{5}$ ${ }^{1}$ Center for Clinical Epidemiology and Methodology, Guangdong Second Provincial General Hospital, Guangzhou, China. ${ }^{2}$ Department of Health Research Methods, Evidence, and Impact, McMaster University, Hamilton, Ontario, Canada. ${ }^{3}$ Information Department, Guangdong Second Provincial General Hospital, Guangzhou, China. ${ }^{4}$ Internet Hospital, Guangdong Second Provincial General Hospital, Guangzhou, China. ${ }^{5}$ Institute for Healthcare Artificial Intelligence Application, Guangdong Second Provincial General Hospital, Guangzhou, China.

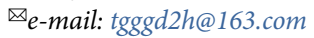

Published online: 20 May 2021

https://doi.org/10.1038/s41591-021-01376-9

References

1. Topol, E. \& Lee, K. F. Nat. Biotechnol. 37, 858-861 (2019).

2. Huang, E. Quartz https://qz.com/1244410/faced-with-a-doctorshortage-a-chinese-hospital-is-betting-big-on-artificialintelligence-to-treat-patients/ (4 April 2018).

3. Luo, P. \& Zhang S. CCTV http.//m news cctvcom/2021/03/02/ ARTIO4HzpGzEscWBIEid8ttH210302.shtml (2 March 2021)

4. Quanlin, Q. China Daily http://www.chinadaily.com. $\mathrm{cn} / \mathrm{a} / 201904 / 01 /$ WS5ca22ffca3104842260b3c56.html (1 April 2019).

5. Yifan, W. China Daily http://www.chinadaily.com.cn/a/201903/ 01/WS5c78f699a3106c65c34ec3a7.html (1 March 2019).

6. Han, Y., Lie, R. K. \& Guo, R. J. Med. Internet Res. 22, el7995 (2020).

7. Koonin, L. M. et al. MMWR Morb. Mortal. Wkly. Rep. 69, 1595-1599 (2020).

8. Li, P. et al. J. Med. Internet Res. 22, e17221 (2020).

9. Ahad, A. et al. Sensors (Basel Switzerland) 20, 4047 (2020).

\section{Acknowledgements}

We acknowledge Z. Ye, Y. Zhou, M. Leng and J. Zhu for help in supporting necessary data and materials for this manuscript.

Competing interests

The authors declare no competing interests. 